\title{
An Exact Penalty Approach for Mixed Integer Nonlinear Programming Problems
}

\author{
Roohollah Aliakbari Shandiz, Nezam Mahdavi-Amiri \\ Faculty of Mathematical Sciences, Sharif University of Technology, Tehran, Iran \\ E-mail:aliakbari_r@mehr.sharif.edu, nezamm@sharif.edu \\ Received July 31, 2011; revised August 19, 2011; accepted September 15, 2011
}

\begin{abstract}
We propose an exact penalty approach for solving mixed integer nonlinear programming (MINLP) problems by converting a general MINLP problem to a finite sequence of nonlinear programming (NLP) problems with only continuous variables. We express conditions of exactness for MINLP problems and show how the exact penalty approach can be extended to constrained problems.
\end{abstract}

Keywords: Mixed Integer Nonlinear Programming, Continuous Programming, Exact Penalty Method, Exact Penalty Functions

\section{Introduction}

One way for relaxing the integer constraints on the variables of a problem is adding an appropriate penalty term to the objective function to create a new problem with only continuous variables. This approach was first introduced by Ragavachari [1] to solve 0-1 linear programming problems and was used by several researchers for solving real nonlinear discrete programming problems [2-5]. Recently, Murray and Ng [6] have extended this approach for large scale 0-1 nonlinear programming problems with linear constraints.

In [7], the exact penalty approach was extended to nonlinear integer programming problems. In $[3,8]$, several penalty functions were presented and the exactness of some of them were proved in [9]. Here, using ideas of Lucidi [9] we introduce conditions for exactness of a penalty function for mixed integer nonlinear programming (MINLP) problems. Then, we extend the exact penalty approach to constrained mixed integer nonlinear programming problems.

Notation 1. Let $v($.) denote the optimal value of problem (.).

\section{Penalty Method for Unconstrained MINLP Problems}

An unconstrained mixed integer nonlinear programming problem is expressed as:

$$
\begin{array}{ccc}
(U M I N L P) & \min & f(x, y) \\
\text { s.t. } & x \in X, y \in Y,
\end{array}
$$

where, $f$ is a real-valued continuous function on $\mathbb{R}^{n+m}, X$ is a finite subset of $\mathbb{Z}^{n}$ in the form $\{0,1\}^{n}$, and $Y$ is a compact subset in $\mathbb{R}^{m}$.

The continuous relaxation of (UMINLP) can be expressed as:

$$
\begin{array}{ccc}
\text { (R) } & \min & f(x, y) \\
& \text { s.t. } & x \in[0,1]^{n}, y \in Y .
\end{array}
$$

We construct the following problem by adding some constraints to the relaxed problem $(R)$ :

$$
\begin{array}{ccc}
\min & f(x, y) \\
(\mathrm{UNLP}) & \text { s.t. } & q(x)=\sum_{i=1}^{n} q_{i}\left(x_{i}\right)=0 \\
& & x \in[0,1]^{n}, y \in Y,
\end{array}
$$

where, the $q_{i}\left(x_{i}\right)$ are nonnegative continuous functions as follows:

$$
q_{i}\left(x_{i}\right)=\left\{\begin{array}{ll}
0, & x_{i} \in\{0,1\}, \\
>0, & x_{i} \in(0,1),
\end{array} \quad i=1, \cdots, n .\right.
$$

It is easy to see that (UMINLP) and (UNLP) are equivalent, because $q(x)=\sum_{i=1}^{n} q_{i}\left(x_{i}\right)$ is zero on points 
in $\{0,1\}^{n}$ and is positive on points in $(0,1)^{n}$.

Some appropriate definitions for the $q_{i}$ are:

(q1) $q_{i}\left(x_{i}\right)=x_{i}\left(1-x_{i}\right), i=1, \cdots, n$,

(q2) $q_{i}\left(x_{i}\right)=1-\cos 2 \pi x_{i}, i=1, \cdots, n$.

Now, for every $r>0$, let

$$
H_{r}(x, y)=f(x, y)+r q(x),
$$

and consider the following penalty problem for the $(U N L P)$ :

$$
\begin{array}{ccc}
\left(U P E N_{r}\right) & \min & H_{r}(x, y)=f(x, y)+r q(x) \\
\text { s.t. } & x \in[0,1]^{n}, y \in Y .
\end{array}
$$

Note that the problem $\left(U P E N_{r}\right)$ is a continuous version of the problem (UMINLP).

Under certain assumptions, we show that for some finite value of penalty parameter $r$, problem $\left(U P E N_{r}\right)$ is equivalent to (UMINLP).

For $\varepsilon, 0<\varepsilon<1 / 2$, define a punctured neighborhood of $\{0,1\}$ in $[0,1]$ as follows:

$$
J_{\varepsilon}=(0, \varepsilon) \cup(1-\varepsilon, 1) .
$$

Assumption 1. There exist $\Delta>0$ and $\gamma>0$ such that i) for every $(x, y) \in J_{\varepsilon}^{n} \times Y \subset[0,1]^{n} \times Y$, we have

$$
\left|\frac{\partial}{\partial x_{i}} f(x, y)\right|<\Delta, i=1, \cdots, n,
$$

ii) each $q_{i}$ is differentiable on $J_{\varepsilon}$ and for each $x_{i} \in J$, we have

$$
q_{i}^{\prime}\left(x_{i}\right)=\left\{\begin{array}{ll}
>\gamma, & x_{i}<\varepsilon, \\
<-\gamma, & x_{i}>1-\varepsilon,
\end{array} \quad i=1, \cdots, n .\right.
$$

Note that if $f$ has bounded derivatives, then it satisfies Assumption 1(i), and as an example, (q1) satisfies Assumption 1(ii).

The following theorem shows that we can find a solution of an unconstrained MINLP problem by solving a finite sequence of NLP problems.

Theorem 1. Under Assumption 1, there exists a finite $r_{0}$ such that for any $r>r_{0}$, any solution of $\left(U P E N_{r}\right)$ also solves (UMINLP) with $v\left(U P E N_{r}\right)=v(U M I N L P)$.

Proof. For any feasible point $(x, y)$ for $(U M I N L P)$, we have

$$
H_{r}(x, y)=f(x, y)+r q(x)=f(x, y) .
$$

Since any feasible point for (UMINLP) is also feasible for $\left(U P E N_{r}\right)$, the above relation implies:

$$
v(U M I N L P) \geq v\left(U P E N_{r}\right) .
$$

For any $r>0$, let $\left(x^{r}, y^{r}\right)$ be an optimal solution of $\left(U P E N_{r}\right)$. Suppose that $\left\{\left(x^{r}, y^{r}\right)\right\}$ is a convergent sub- sequence of optimal solutions of $\left(U P E N_{r}\right)$ and $\left(x^{*}, y^{*}\right)$ is its limit. Note that since $\left\{\left(x^{r}, y^{r}\right)\right\} \subset[0,1]^{n} \times Y$ and $[0,1]^{n} \times Y$ is compact, at least one convergent subsequence exists.

Since $x^{r} \rightarrow x^{*}$, there exists an $\bar{r}$ such that for every $r>\bar{r}$, we have: $\left\|x^{r}-x^{*}\right\|_{\infty}<\varepsilon$. Therefore, (2) implies that $x_{i}^{r} \in\{0,1\}$ or $x_{i}^{r} \in J_{\varepsilon}$.

Now, let $r_{0}=\max (\bar{r}, \Delta / \gamma)$ and suppose that $r>r_{0}$. If $x_{i}^{r} \notin\{0,1\}$, then $x_{i}^{r} \in J_{\varepsilon}$. Since $f$ and $q_{i}$ are differentiable on $J_{\varepsilon}$ and the first-order necessary conditions for problem $\left(U P E N_{r}\right)$ holds in subspace $J_{\varepsilon}$, then we have

$$
\begin{gathered}
\frac{\partial}{\partial x_{i}} H_{r}\left(x^{r}, y^{r}\right)=0 \\
\frac{\partial}{\partial x_{i}} f\left(x^{r}, y^{r}\right)+r \frac{\partial}{\partial x_{i}} q\left(x^{r}, y^{r}\right)=0, \\
\frac{\partial}{\partial x_{i}} f\left(x^{r}, y^{r}\right)+r \frac{\partial}{\partial x_{i}} q_{i}\left(x_{i}^{r}\right)=0 .
\end{gathered}
$$

Assumption 1(ii) implies:

$$
\left|\frac{\partial}{\partial x_{i}} f\left(x^{r}, y^{r}\right)\right|=r\left|q_{i}^{\prime}\left(x_{i}^{r}\right)\right|>\frac{\Delta}{\gamma} \times \gamma=\Delta .
$$

This is clearly a contradiction to Assumption 1(i). Therefore, for $r>r_{0}$, we have $x^{r} \in\{0,1\}^{n}$. Thus, $\left(x^{r}, y^{r}\right)$ is feasible for (UMINLP) and $q\left(x^{r}\right)=0$. Furthermore,

$$
\begin{aligned}
v\left(\text { UPEN }_{r}\right) & =H_{r}\left(x^{r}, y^{r}\right) \\
& =f\left(x^{r}, y^{r}\right)+r q\left(x^{r}\right) \\
& =f\left(x^{r}, y^{r}\right) \\
& \geq v(\text { UMINLP }) .
\end{aligned}
$$

Relations (3) and (4) imply:

$$
\begin{aligned}
f\left(x^{r}, y^{r}\right) & =v(\text { UMINLP })=v\left(\text { UPEN }_{r}\right) \\
& =H_{r}\left(x^{r}, y^{r}\right) .
\end{aligned}
$$

Therefore, for any $r>r_{0},\left(x^{r}, y^{r}\right)$ is an optimal solution for both problems.

\section{Exact Penalty Functions}

The following penalty functions have been suggested [3, 9] for zero-one problems ( $\left.0 \leq x_{i} \leq 1\right)$ :

$$
\text { (q3) } q_{i}\left(x_{i}\right)=4 x_{i}\left(1-x_{i}\right)
$$

$$
\text { (q4) } q_{i}\left(x_{i}\right)= \begin{cases}2 x_{i}, & x_{i} \leq \frac{1}{2}, \\ 2\left(1-x_{i}\right), & x_{i}>\frac{1}{2},\end{cases}
$$




$$
\begin{aligned}
& \text { (q5) } q_{i}\left(x_{i}\right)=\log \left(x_{i}+\theta\right)+\log \left(1-x_{i}+\theta\right) \\
& -\log \theta-\log (1+\theta) \text {, } \\
& \text { (q6) } q_{i}\left(x_{i}\right)=-\left(x_{i}+\theta\right)^{-p}-\left[\left(1-x_{i}\right)+\theta\right]^{-p} \\
& +\theta^{-p}+(1+\theta)^{-p} \\
& \text { (q7) } q_{i}\left(x_{i}\right)=1-\exp \left(-\alpha x_{i}\right) \\
& +1-\exp \left(-\alpha\left(1-x_{i}\right)\right)-1-\exp (-\alpha), \\
& \text { (q8) } q_{i}\left(x_{i}\right)=x_{i}^{q}+\left(1-x_{i}\right)^{q}-1 \text {, } \\
& \text { (q9) } q_{i}\left(x_{i}\right)=\left[1+\exp \left(-\alpha x_{i}\right)\right]^{-1} \\
& +\left[1+\exp \left(-\alpha\left(1-x_{i}\right)\right)\right]^{-1} \\
& -\frac{1}{2}-1-\exp (-\alpha)^{-1} \text {, }
\end{aligned}
$$

where, $\alpha, p>0,0<\theta<1 / 2$ and $0<q<1$. Penalty functions $(q 5)-(q 9)$ were introduced in [9]. Here, to have (1) satisfied, we add a fixed number to every function $(q 5)-(q 9)$.

Also, two other penalty functions for zero-one problems can be defined as follows:

$$
\begin{gathered}
(q 10) \quad q_{i}\left(x_{i}\right)=\sin \left(\pi x_{i}\right), \\
(q 11) \quad q_{i}\left(x_{i}\right)=x_{i}\left(1-x_{i}\right)\left(x_{i}+\tau\right)\left(1-x_{i}+\tau\right),
\end{gathered}
$$

where, $\tau>0$.

Note that any bounded MINLP problem can be reformulated as a mixed zero-one programming problem by using the following representation for the integer variables (see [7]):

$$
x_{i}=\sum_{k=0}^{M} 2^{k} y_{k}^{(i)}, y_{k}^{(i)} \in\{0,1\}, i=1, \cdots, n,
$$

where, $M$ is an upper integer bound for $\log x_{i}$. Thus, we can use the penalty functions for all bounded integer problems.

Also, note that direct use of penalty functions for MINLP problems (not zero-one) is not suitable, because due to the structure of the $q_{i}$ (see (1)), the resulting nonconvex optimization problem, in general, may have many local minimizers (see [4]).

Now, we show that $\left(U P E N_{r}\right)$ with the penalty functions $(q 3)-(q 11)$ are exact for (UMINLP). Note that exactness of $(q 5)-(q 9)$ have already been proved in [9]. Here, by using Theorem 1, we prove the exactness corresponding to all of $(q 3)-(q 11)$.

Let us suppose that $f$ satisfies Assumption 11 ). We then need to show that Assumption 12 ) holds for every one of $(q 3)-(q 11)$. For $\varepsilon=1 / 3$, we have $J_{\varepsilon}=(0,1 / 3) \cup(2 / 3,1)$. It is easy to show that for the functions $(q 3)-(q 11)$, we have

$$
\forall x_{i} \in\left(0, \frac{1}{3}\right), q_{i}^{\prime}\left(x_{i}\right)>q_{i}^{\prime}(1 / 3)>0,
$$

and

$$
\forall x_{i} \in\left(\frac{2}{3}, 1\right), q_{i}^{\prime}\left(x_{i}\right)<q_{i}^{\prime}(2 / 3)<0 .
$$

Therefore, Assumption 1(ii) is satisfied for $(q 3)-(q 11)$. Thus, the penalty problem $\left(U P E N_{r}\right)$. with any one of the functions $(q 3)-(q 11)$ is exact for (UMINLP).

\section{Extension to Constrained Problems}

A constrained mixed integer nonlinear programming problem is expressed as:

$$
\begin{array}{ccc} 
& \min & f(x, y) \\
(M I N L P) & \text { s.t. } & g_{j}(x, y) \leq 0, j=1, \cdots, l, \\
& & x \in\{0,1\}^{n}, y \in Y,
\end{array}
$$

where, $Y$ is a compact subset in $\mathbb{R}^{m}$.

$$
\text { Let } S=\left\{(x, y) \in\{0,1\}^{n} \times Y \mid g_{j}(x, y) \leq 0, j=1, \cdots, l\right\} \text {. }
$$

A penalty function for (MINLP) is defined as follows:

$$
p(x, y)= \begin{cases}0, & (x, y) \in S \\ >0, & (x, y) \notin S .\end{cases}
$$

A typical penalty function for the constraints $g_{j}$ in $(M I N L P)$ is:

$$
p(x, y)=\sum_{j=1}^{l} \max \left(0, g_{j}(x, y)\right) .
$$

Consider the penalty problem of $(M I N L P)$ as:

$$
\begin{array}{ccc}
\left(P E N_{\mu}\right) & \min & H_{\mu}(x, y)=f(x, y)+\mu p(x, y) \\
\text { s.t. } & x \in\{0,1\}^{n}, y \in Y,
\end{array}
$$

and define the following continuous penalty problem for $(M I N L P)$ :

$$
\begin{aligned}
& \left(P E N_{\mu, r}\right) \quad \min \quad \begin{aligned}
H_{\mu, r}(x, y)= & f(x, y)+\mu p(x, y) \\
&
\end{aligned} \\
& \text { s.t. } \quad x \in[0,1]^{n}, y \in Y \text {. }
\end{aligned}
$$

To prove the exactness of $\left(P E N_{\mu, r}\right)$ for $(M I N L P)$, first we show that for some penalty function $p,\left(P E N_{\mu}\right)$ is exact for $(M I N L P)$. Note that exactness for some penalty functions, such as absolute-value penalty function, for the nonlinear programming $(N L P)$ problems or nonlinear integer programming (NLIP) problems has already been proved (see $[10,11]$ ), that is, it has been shown that for a finite value of the penalty parameter, the main problem and the corresponding penalty problem are equivalent. Here, we prove exactness for the constrained 
MINLP problems.

Theorem 2. Consider (MINLP) and for every $x$, define the following NLP problem,

$$
\begin{array}{ccc} 
& \min & f(x, y) \\
\left(N L P^{x}\right) & \text { s.t. } & g_{j}(x, y) \leq 0, j=1, \cdots, l, \\
& & y \in Y,
\end{array}
$$

and its corresponding penalty problem,

$$
\begin{array}{ccc}
\left(P E N_{\mu}^{x}\right) & \min & H_{\mu}(x, y)=f(x, y)+\mu p(x, y) \\
\text { s.t. } & y \in Y .
\end{array}
$$

Suppose that for any $x$ feasible to $\left(N L P^{x}\right)$, there exists a $\mu_{x}$ such that for every $\mu>\mu_{x}$, problems $\left(N L P^{x}\right)$ and $\left(P E N^{x}\right)$ are equivalent. Then, there exists a $\mu_{0}$ such that for every $\mu>\mu_{0}$, any solution of $\left(P E N_{\mu}\right)$ also solves $(M I N L P)$ and $v\left(\right.$ PEN $\left._{\mu}\right)=v($ MINLP $)$.

Proof. For any feasible point $(x, y)$ of $(M I N L P)$, we have

$$
H_{\mu}(x, y)=f(x, y)+\mu p(x, y)=f(x, y) .
$$

Since any feasible point for (MINLP) is also feasible for $\left(P E N_{\mu}\right)$, it follows from the above equality that

$$
v(M I N L P) \geq v\left(P E N_{\mu}\right) .
$$

We know:

$$
\begin{aligned}
v\left(\text { PEN }_{\mu}\right) & =\min _{(x, y) \in\{0,1\}^{n} \times Y} H_{\mu}(x, y) \\
& =\min _{(x, y) \in\{0,1\}^{n} \times Y} f(x, y)+\mu p(x, y) \\
& =\min _{x \in\{0,1\}^{n}} \min _{y \in Y} f(x, y)+\mu p(x, y) .
\end{aligned}
$$

For any fixed $x$, define

$$
S_{x}=\left\{y \in Y \mid g_{j}(x, y) \leq 0, j=1, \cdots, l\right\} .
$$

Consider the following two cases.

Case 1: $S_{x} \neq \varnothing$. Consider the following problem:

$$
\min _{y \in Y} f(x, y)+\mu p(x, y) .
$$

From the assumption of the theorem, there exists $\mu_{x}$ such that for any $\mu>\mu_{x}$, any solution of the above problem is also a solution of the following problem,

$$
\min _{y \in S_{x}} f(x, y) .
$$

Case 2: $S_{x}=\varnothing$. From the definition of $S_{x}$, for any $y \in Y$, we have $p(x, y)>0$. Since $Y$ is compact, then

$$
\min _{y \in Y} p(x, y)=\delta_{x}>0 .
$$

Let $f$ be a lower bound of $f$ on $\{0,1\}^{n} \times Y$, and $\mu_{x}=(v(M I N L P)-\underline{f}+1) / \delta_{x}$. For any $\mu>\mu_{x}$, we have

$$
\begin{aligned}
f(x, y)+\mu p(x, y) & >f(x, y)+\mu_{x} p(x, y) \\
& \geq \underline{f}+\mu_{x} \delta_{x}=v(M I N L P)+1 .
\end{aligned}
$$

This means that if $\mu>\mu_{x}$, then $f(x, y)+\mu p(x, y)>v(M I N L P)+1$. Thus, (6) implies that if $\mu>\mu_{x}$, then minimum does not occur in this case. Now, let $\mu_{0}=\max _{x \in\{0,1\}} \mu_{x}$. For any $\mu>\mu_{0}$, if $\left(x^{*}, y^{*}\right)$ is an optimal solution of $\left(P E N_{\mu}\right)$, then from the previous implication we get that Case 2 does not occur. From Case 1, we have

$$
\begin{aligned}
f\left(x^{*}, y^{*}\right)+\mu p\left(x^{*}, y^{*}\right) & =v\left(P E N_{\mu}\right) \\
& =\min _{x \in\{0,1\}^{n}} \min _{y \in Y} f(x, y)+\mu p(x, y) \\
& =\min _{x \in\{0,1\}^{n}} \min _{y \in S_{x}} f(x, y) .
\end{aligned}
$$

Let $\bar{f}$ be an upper bound of $f$ on $\{0,1\}^{n} \times Y$. Then

$$
f\left(x^{*}, y^{*}\right)+\mu p\left(x^{*}, y^{*}\right) \leq \bar{f} .
$$

Since this relation holds for any $\mu>\mu_{0}$, we have $p\left(x^{*}, y^{*}\right)=0$. Therefore, $\left(x^{*}, y^{*}\right)$ is feasible to (MINLP). Furthermore,

$$
\begin{aligned}
f\left(x^{*}, y^{*}\right) & =v\left(P E N_{\mu}\right) \\
& =\min _{x \in\{0,1\}^{n}} \min _{y \in S_{x}} f(x, y) \\
& =\min _{x \in\{0,1\}^{n}, y \in Y, g_{j}(x, y) \leq 0} f(x, y) \\
& =v(\operatorname{MINLP}) .
\end{aligned}
$$

Thus, $\left(x^{*}, y^{*}\right)$ is an optimal solution for both $\left(P E N_{\mu}\right)$ and (MINLP).

Theorem 2 shows that if $p$ is an exact penalty function for an NLP problem, then it is also exact for the MINLP problem. Thus, (5) is an exact penalty function for (MINLP).

Now, we show that $\left(P E N_{\mu, r}\right)$ is exact for $(M I N L P)$, that is, for finite penalty parameter values of $r$ and $\mu$, $\left(P E N_{\mu, r}\right)$ and $(M I N L P)$ are equivalent.

Assumption 2. Assumption 1(i) holds for each $g_{j}$, namely, for each $(x, y) \in J_{\varepsilon}^{n} \times Y$, we have

$$
\left|\frac{\partial}{\partial x_{i}} g_{j}(x, y)\right|<\Delta, i=1, \cdots, n, j=1, \cdots, l .
$$

Theorem 3. Suppose that both Assumption 1 and Assumption hold and $p$ is an exact penalty function for (MINLP). Then, there exist $r_{0}$ and $\mu_{0}$ such that for every $r>r_{0}$ and $\mu>\mu_{0}$, any solution of $\left(P E N_{\mu, r}\right)$ also solves $(M I N L P)$ and $v\left(P E N_{\mu, r}\right)=v(M I N L P)$.

Proof. Since $p$ is an exact penalty function for (MINLP), there exists a $\mu_{0}$ such that for each $\mu>\mu_{0}$, any solution for $\left(P E N_{\mu, r}\right)$ also solves (MINLP) and 
$v\left(P E N_{\mu}\right)=v(M I N L P)$.

Theorem 1 on $\left(P E N_{\mu, r}\right)$ implies that there exists an $r_{0}$ such that for every $r>r_{0}$, any solution of $\left(P E N_{\mu, r}\right)$ is also a solution of $\left(P E N_{\mu}\right)$ and $v\left(P E N_{\mu, r}\right)=v\left(P E N_{\mu}\right)$ Therefore, for every $\mu>\mu_{0}$ and $r>r_{0}$, any solution of $\left(P E N_{\mu, r}\right)$ also solves $(M I N L P)$ and $v\left(P_{\mu N}, r\right)=v(M I N L P)$.

\section{Summary}

We proposed an exact penalty approach for solving mixed integer nonlinear programming (MINLP) problems and showed how to convert a MINLP problem to a finite sequence of NLP problems. We stated conditions for exactness of a penalty function for MINLP problems and showed how exact penalty functions for NLP problems could serve as exact penalty functions for MINLP problems.

\section{Acknowledgements}

The second author thanks the support of Research Council of Sharif University of Technology.

\section{References}

[1] M. Ragavachari, "On Connections between Zero-One Integer Programming and Concave Programming under Linear Constraints," Operations Research, Vol. 17, No. 4, 1969, pp. 680-684. doi:10.1287/opre.17.4.680

[2] J. Cai and G. Thierauf, "Discrete Optimization of Structures Using an Improved Penalty Function Method," Engineering Optimization, Vol. 21, No. 4, 1993, pp. 293-306. doi:10.1080/03052159308940981

[3] J. F. Fu, R. G. Fenton and W. L. Cleghorn, "A Mixed Integer-Discrete-Continuous Programming Method and Its Application to Engineering Design Optimization," Engineering Optimization, Vol. 17, No. 4, 1991, pp. 263280. doi:10.1080/03052159108941075

[4] S. S. Rao, "Engineering Optimization: Theory and Practice," 4th Edition, Wiley, Hoboken, 2009.

[5] D. K. Shin, Z. Gürdal and O. H. Griffin Jr., "A Penalty Approach for Nonlinear Optimization with Discrete Design Variables," Engineering Optimization, Vol. 16, No. 1, 1990, pp. 29-42.doi:10.1080/03052159008941163

[6] W. Murray and K. M. Ng, "An Algorithm for Nonlinear Optimization Problems with Binary Variables," Computational Optimization and Applications, Vol. 47, No. 2, 2008, pp. 257-288. doi:10.1007/s10589-008-9218-1

[7] F. Giannessi and F. Niccolucci, "Connections between Nonlinear and Integer Programming Problems," Symposia Mathematica, Vol. 19, 1976, pp. 161-176.

[8] F. Rinaldi, "New Results on the Equivalence between Zero-One Programming and Continuous Concave Programming," Optimation Letters, Vol. 3, No. 3, 2009, pp. 377-386. doi:10.1007/s11590-009-0117-x

[9] S. Lucidi and F. Rinaldi, "Exact Penalty Functions for Nonlinear Integer Programming Problems," Journal of Optimization Theory and Applications, Vol. 145, No. 3, 2010, pp. 479-488. doi:10.1007/s10957-010-9700-7

[10] D. Li and X. Sun, "Nonlinear Integer Programming," Springer, New York, 2006.

[11] D. G. Luenberger and Y. Ye, "Linear and Nonlinear Programming," 3rd Edition, Springer, New York, 2008. 